Pacific Journal of Mathematics

ON PERMUTATIONS INDUCED BY LINEAR VALUE
FUNCTIONS 


\title{
ON PERMUTATIONS INDUCED BY LINEAR \\ VALUE FUNCTIONS
}

\author{
Stewart S. CAIRNS
}

1. Background and statement of the problem. Consider a set of $n$ objects, symbolized by the integers

$$
N=(1, \cdots, n) \text {. }
$$

Let $v_{i}$ be a real number to be called the value of object $i(i=1, \cdots, n)$. The value of $J=\left(i_{1}, \cdots, i_{j}\right) \subset N$ will mean

$$
v(J)=\sum_{h=1}^{j} v_{i_{h}},
$$

and the value of the null set $J_{0}$ is

$$
v\left(J_{0}\right)=0
$$

The present paper is partly motivated by its bearing on linear programming problems in which a subset of $N$ is sought, having a maximum value among all subsets satisfying some given restriction; for example, a condition of the form $\sum_{h=1}^{j} w_{i_{h}}<W$. In this restriction, $w$ might be the weight of object $i$, and one would be seeking a subset of maximum value among those with a given upper bound on their total weights. In many applications, $v_{i}$ and $w_{i}$ are positive, but we do not impose this condition at present.

Let $\{J\}$ be the set of all the $2^{n}$ subsets of $N$. Given $J \in\{J\}$, we will denote with $[J]$ the set of all subsets of $N$ each having the same value as $J$. Thus $\{J\}$ is partitioned into equivalence classes, each of the form

$$
[J]=\{K \subset N \mid v(K)=v(J)\} .
$$

These equivalence classes are ordered by the relation $\prec$, to be read precedes, defined thus:

$$
[J] \prec[K] \text { if } v(J)<v(K) .
$$

(A) We will denote by $I I(V ;[J])$ the permutation of the equivalence classes in which they are arranged in order of increasing values; that is, $[J]$ comes before $[K]$ if $[J] \prec[K]$.

Received September 28, 1961. This is a revision of a paper written in 1955 at the RAND Corporation (RAND No. P-735) and presented to the American Mathematical Society, December, 1955. The title, before revison, was "On the Partition of the Vertices of an $n$-Cube by an $(n-1)$-Plane." 
(B) There exists only one equivalence class, $[J]=\{J\}$, if and only if $v_{i}=0(i=1, \cdots, n)$. Hereafter, we exclude this trivial case from consideration and assume ${ }^{1}$

$$
\Sigma\left|v_{i}\right|>0 \text {. }
$$

We next define a geometric interpretation. Let $\{p\}$ be the set of vertices of the unit cube

$$
C: 0 \leqq x_{i} \leqq 1 \quad(i=1, \cdots, n)
$$

in a euclidean $n$-space $E^{n}$ with coordinate system $\left(x_{1}, \cdots, x_{n}\right)$. With each $J \in\{J\}$, we associate the vertex $p(J)$ with coordinates

$$
x_{i}(p(J))=\left\{\begin{array}{lll}
1 & \text { if } & i \in J \\
0 & \text { if } & i \notin J
\end{array} .\right.
$$

Conversely, we denote with $J(p)$ the subset of $N$ defined by

$$
J(p)=\left\{i \mid x_{i}=1 \text { at } p, p \in\{p\}\right\},
$$

(C) There is thus a one-to-one mapping $J \rightarrow p(J)$, with inverse $p \rightarrow J(p)$, of the $2^{n}$ subsets $\{J\}$ of $N$ onto the $2^{n}$ vertices $\{p\}$ of $C$. The value of $J(p)$ equals the value of $\sum v_{i} x_{i}$ at $p$.

Condition (1.6) implies that $V$ determines a 1-parameter family of parallel hyperplanes

$$
L^{n-1}(c): \sum v_{i} x_{i}=c
$$

which fiber $E^{n}$.

For each vertex $p \in\{p\}$, let $v(p)$ denote the value such that $p \in L^{n-1}(v(p))$. We refer to $v(p)$ as the value of $p$. Let

$$
[q]=\{p \in\{p\} \mid v(p)=v(q)\} \text {. }
$$

Thus $\{p\}$ is partitioned into equivalence classes $[q]$, which are the images of the classes $[J]$ under the mapping of $\{J\} \rightarrow\{p\}$ which takes $J$ into $p(J)$. We define $\prec$ for these classes of vertices by

$$
[p] \prec[q] \text { if } v(p)<v(q),
$$

and we denote by $\Pi(V ;[p])$ the permutation defined exactly as in (A) with $p, q$ replacing $J, K$.

(D) Thus a study of the permutations $\Pi(V ;[p])$ is entirely equivalent to a study of the permutations $\|(V ;[J])$. Geometrically, $[p]$ is the set of all vertices of $C$ on the hyperplane $L^{n-1}(v(p))$; and $\Pi(V ;[p])$ is the arrangement of the classes $[p]$ in the order in which the hyperplane

\footnotetext{
1 Throughout this paper, $\sum_{i=1}^{n}$ is abbreviated to $\sum$.
} 
$L^{n-1}(c)$ passes through them as the parameter $c$ increases from a value less than $\min v(p)$ to a value exceeding $\max v(p)$.

2. Some simplifying hypotheses. We will refer to $V=\left(v_{1}, \cdots, v_{n}\right)$, in the above notation, as a value set, and to the function $v$ on $\{J\}$, defined by (1.2), as the corresponding value function.

If $i$ is not in the subset $J=\left(i_{1}, \cdots, i_{j}\right)$ of $N$, then $\left(i, i_{1}, \cdots, i_{j}\right) \in[J]$ if and only if $v_{i}=0$.

(A) We assume hereafter that $v_{i} \neq 0(i=1, \cdots, n)$, since the foregoing statement makes it easy to extend our results to cases where some $v$ 's equal zero.

Let $\left(v_{i_{1}}, \cdots, v_{i_{g}}\right)$ be the positive and $\left(v_{h_{1}}, \cdots, v_{h_{k}}\right)$ the negative numbers in the value set $V=\left(v_{1}, \cdots, v_{n}\right)$. Then

$$
\begin{aligned}
& \min v(p)=\sum_{r=1}^{k} v_{h_{r}}=\min v(J)=v\left(h_{1}, \cdots, h_{k}\right) \\
& \max v(p)=\sum_{s=1}^{j} v_{i_{s}}=\max v(J)=v\left(i_{1}, \cdots, i_{j}\right) .
\end{aligned}
$$

Furthermore, $v(p)$ assumes its minimum only at the point where $x_{h_{r}}=1, x_{i_{s}}=0(r=1, \cdots, k ; s=1, \cdots, j)$, and its maximum only at the diametrically opposite vertex, where $x_{h_{r}}=0$ and $x_{i_{s}}=1$.

Consider the transformation from $\left(x_{1}, \cdots, x_{n}\right)$ to a coordinate system $\left(y_{1}, \cdots, y_{n}\right)$ defined by

$$
\begin{array}{ll}
y_{h_{r}}=1-x_{h_{r}} & (r=1, \cdots, k) \\
y_{i_{s}}=x_{i_{s}} & (s=1, \cdots, j) .
\end{array}
$$

In terms of the new system, the hyperplane $L^{n-1}(c)$ is defined by

$$
\sum w_{i} y_{i}=d
$$

where

$$
\begin{aligned}
w_{h_{r}} & =-v_{h_{r}} & (r=1, \cdots, k) \\
w_{i_{s}} & =v_{i_{s}} & (s=1, \cdots, j) \\
d & =c-\sum_{r=1}^{k} v_{h_{r}} . &
\end{aligned}
$$

Hence, if we know the permutation $\Pi(W,[J])$, we can deduce from it the permutation $\Pi(V,[J])$, as follows: The $t$ th element $\left[J_{t}\right]$ of $\Pi(V,[J])$ is obtained from the $t$ th element, $\left[K_{t}\right]$ of $\Pi(W,[J])$ by the formula

$$
K_{t}=\left(J_{t} \cap\left(i_{1}, \cdots, i_{j}\right)\right) \cup\left(\left(h_{1}, \cdots, h_{k}\right)-J_{t} \cap\left(h_{1}, \cdots, h_{k}\right)\right) .
$$


(B) Thus the problem of finding an arbitrary $\Pi(V,[J])$ is reduced to the case where $v_{i}>0(i=1, \cdots, n)$, a condition hereafter assumed.

LEMma 2.1. Let $V=\left(v_{1}, \cdots, v_{n}\right)$ be an arbitrary value set, and let $\delta>0$ be arbitrarily small. Then there exists a value set $W=$ $\left(w_{1}, \cdots, w_{n}\right)$, such that $\left|w_{i}-v_{i}\right|<\delta$, and that $W$ assigns $2^{n}$ different values to the $2^{n}$ different subsets $\{J\}$ of $N$.

Proof. Hypothesis: For some $k>0$, there exist real numbers $\left(w_{1}, \cdots, w_{k-1}\right)$ such that the sums $\left(w_{i_{1}}+\cdots+w_{i_{j}}\right)$, as $\left(i_{1}, \cdots, i_{j}\right)$ ranges over all $2^{k-1}$ subsets of $(1, \cdots, k-1)$, are all different, where the sum is defined as zero when $\left(i_{1}, \cdots, i_{j}\right)$ is the vacuous set.

Now consider the numbers $\sigma_{i}\left(i=1, \cdots, 2^{k}\right)$ consisting of these $2^{k-1}$ sums and the $2^{k-1}$ numbers obtained by adding $v_{k}$ to each of them. If the $\sigma_{i}$ are all different, let $w_{k}=v_{k}$. If not, then let $w_{k}$ be chosen so that $0<\left|w_{k}-v_{k}\right|<\delta$, and that $\left|w_{k}-v_{k}\right|$ is less than the smallest nonzero difference $\left|\sigma_{j}-\sigma_{i}\right|$. Then $\left(w_{1}, \cdots, w_{k}\right)$ is easily seen to satisfy the condition in the hypothesis with $k+1$ in place of $k$.

This completes Step $k$ of an inductive argument. In Step 1, $\left(w_{1}, \cdots, w_{k-1}\right)$ is vacuous, and the hypothesis is trivially fulfilled. Step $n$ yields the lemma.

The condition $\left|w_{i}-v_{i}\right|<\delta$ implies, by (1.2), $|w(J)-v(J)|<j \delta$, where $j$ is the number of objects in $J$. Hence, for each $J \in\{J\}$, $|w(J)-v(J)|<n \delta$. Now, let $\eta$ equal the smallest non zero difference of the form $|v(J)-v(K)|$ as $(J, K)$ range over all pairs of subsets of $N$, and let $\delta$ be less than $\eta / 4 n$. Then,

(a) $v(J) \neq v(K) \Rightarrow|v(J)-v(K)| \geqq \eta \Rightarrow|w(J)-w(K)|>\eta / 2$.

(b) $v(J)=v(K) \Rightarrow|v(J)-v(K)|=0 \Rightarrow|w(J)-w(K)|<\eta / 2$.

Otherwise expressed, given $J \in\{J\}$, the equivalence class $[J]$ consists of all those subsets $K$ of $N$ for which $|w(J)-w(K)|<\eta / 2$. In the permutation of $\{J\}$ determined by $W$, these elements are consecutive, since they clearly can not be separated by elements whose $w$-values differ by more than $\eta / 2$ from $w(J)$.

(C) This motivates the assumption, which we hereafter make, that $v(J) \neq v(K)$ if $J \neq K$. For, Lemma 2.1 permits us to shift to this case from the general case by an arbitrarily small perturbation of the value function, after which we can shift back by amalgamating into equivalence classes those elements of $\{J\}$ whose $w$-values are almost equal, in the sense of $(2.6 b)$.

(D) Finally, we assume $v_{1}<\cdots<v_{n}$. The general case can be 
solved with the aid of this one by (1) renumbering the objects in the set $N$ in order of increasing values, (2) finding the corresponding permutation, then (3) returning to the original numbering to obtain $I I(V,[J])$.

(E) Combining (A), (B), (C) and (D), we assume hereafter that

$$
0<v_{1}<\cdots<v_{n}
$$

and that $v(J) \neq v(K)$ if $J \neq K$. This implies that each equivalence class $[J]$ consists of a single subset $J$ of $N$. We accordingly write, hereafter, $\Pi(V, J)$ instead of $\Pi(V,[J])$.

3. Some properties of special value permutations. A special value permutation will mean a permutation $\Pi(V, J)$, or $\Pi(V, p)$, subject to $\S 2(\mathrm{E})$. The set of all special value permutations will be denoted by $\{I I\}$.

(A) Let $J_{0}$ be the vacuous set and $J_{2^{n-1}}$ the entire set $N=(1, \cdots, n)$. Correspondingly, let $p_{0}$ be the origin and $p_{2^{n-1}}$ the point $(1, \cdots, 1)$. Then, each $\Pi(V, J)$ or $\Pi(V, p)$ commences with $J_{0}$ or $p_{0}$ and terminates with $J_{2^{n}-1}$ or $p_{2^{n}-1}$.

(B) For $c$ real, let

$$
\begin{aligned}
\{p\}_{c} & =\{p \mid v(p)<c\} \\
\{J\}_{c} & =\{J \mid v(J)<c\} .
\end{aligned}
$$

Then, (1) $\{p\}_{0}$ and $\{J\}_{0}$ are vacuous, and (2) as $c$ increases from 0 to $1+\sum v_{i}$, the elements of $\{p\}$ and of $\{J\}$ are adjoined to $\{p\}_{c}$ and to $\{J\}_{c}$ in the order of their occurrence in $\Pi(V, p)$ and $\Pi\{V, J\}$ respectively.

(C) Convention. A nonvacuous subset $J \in\{J\}$ of $N$ will sometimes be denoted by its elements, juxtaposed and written in order of increasing magnitude, thus;

$$
\left.J=i_{1} \cdots i_{j} \text { where } 0<i_{1}<\cdots<i_{j} \leqq n\right) .
$$

The vacuous subset will be denoted by $J_{0}=0$. These symbols could also be interpreted as representing integers in the number system with radix $n+1$, to be called the $(n+1)$-system. When so interpreted, $J$ will be modified by a star, thus:

$$
\begin{aligned}
& J^{*}=i_{1} \cdots i_{j}=\sum_{h=1}^{j} i_{h}(n+1)^{j-h} \\
& J_{0}^{*}=0
\end{aligned}
$$

The use of $i_{1} \cdots i_{j}$ for both $J$ and $J^{*}$ should cause no confusion, since 
$\Pi(V, J)$ could be interpreted as a permutation $\Pi\left(V, J^{*}\right)$ of the $2^{n}$ numbers $\left\{J^{*}\right\}=\left\{J^{*} \mid J \in\{J\}\right\}$.

LEMMA 3.1. Let $\left(J_{0}^{*}, \cdots, J_{2^{n}-1}^{*}\right)$ be the numbers $\left\{J^{*}\right\}$ arranged in order of increasing magnitude. The special value set $V_{0}=\left(v_{1}, \cdots, v_{n}\right)$ defined by

$$
\begin{array}{rlr}
v_{j} & =\sum_{i=1}^{j}(n+1)^{n-i} \quad \quad(j=1, \cdots, n) \\
& =\overbrace{11 \cdots 1}^{j \text { digits }} n \overbrace{0 \cdots 0}^{n-j \text { digits }} \quad(\text { in the }(n+1) \text {-system })
\end{array}
$$

yields the permutation

$$
\Pi_{0}(J)=\Pi\left(V_{0}, J\right)=J_{0}, J_{1}, \cdots, J_{2^{n-1}} .
$$

For example, if $n=4$,

$$
\Pi_{\mathrm{o}}(J)=0,1,2,3,4,12,13,14,23,24,34,123,124,134,234,1234
$$

Proof. Let $J=j_{1} \cdots j_{h}$ be an element of $\{J\}$. Then (see (B) above) $J$ joins $\{J\}_{c}$ as $c$ increases through the value

$$
\begin{aligned}
& v(J)=\sum_{i \in J} v_{i}= \\
& \overbrace{h h \cdots h}^{\left(j_{1}\right)} \overbrace{(h-1) \cdots(h-1)}^{\left(j_{2}-j_{1}\right)} \overbrace{(h-2) \cdots(h-2)}^{\left(j_{3}-j_{2}\right)} \ldots \overbrace{11 \cdots 1}^{\left(j_{h}-j_{h-1}\right)\left(n-j_{h}\right)} \overbrace{0 \cdots 0}
\end{aligned}
$$

in the $(n+1)$-system. To complete the proof, it is sufficient to show that $v(J)>v(K)$ if and only if $J^{*}>K^{*}$. To see this, first note that the initial digit of $v(J)$ equals the number of elements in $J$, so that $v(J)>v(K)$ if $J$ contains more elements than $K$. If $J$ and $K$ contain the same number $h$ of elements, then $v(J)$ and $v(K)$ both have $h$ for initial digit. If, in addition, $J^{*}>K^{*}$ then it is easily seen that the first corresponding unequal digits of $v(J)$ and $v(K)$ are of the forms $h-i$ and $h-i-1$, respectively. The proof is easy to complete.

Lemma 3.2. Let $J, H, K$ be elements of $\{J\}$. Then, in $\Pi(V, J)$, subject to $\S 2(\mathrm{E})$

(a) $J \prec J \cup K \quad$ unless $K \subset J$

(b) $J \prec K \Longleftrightarrow J \cup H \prec K \cup H$ if $J \cap H=K \cap H=J_{0}$

(c) $J \prec K \Rightarrow J \cup H \prec K \cup H$ if $J \cap H \supset K \cap H$

(d) $J \prec K \Longleftrightarrow K^{\prime} \prec J^{\prime}$ where $J^{\prime}$ and $K^{\prime}$ are the respective com. plements of $J$ and $K$ in $N$.

(e) $j_{i} \leqq k_{i}(i=1, \cdots, h) \Rightarrow\left(j_{1}, \cdots, j_{h}\right) \prec\left(k_{1}, \cdots, k_{h}\right)$, if $j_{i}<k_{i}$ for some $i$. 
Proof. Parts (a), (b), (c) and (d) follow from $v_{i}>0$. Part (e) is a consequence of (2.7).

To illustrate the lemma, note that $(1,3,6) \prec(1,2,3,6,7)$ by part (a). By part (e), $(1,3,6) \prec(2,3,7) \prec(3,4,9)$. Since $(2,3) \prec(4,5)$ by part (e), it follows from part (a) and the transitivity of the relationship $\prec$ that $(2,3) \prec(1,4,5)$. Also if, in some $\Pi(V, J),(1,4,6) \prec(1,3,7)$, then, by $(\mathrm{b}),(4,6) \prec(3,7),(2,4,6) \prec(2,3,7)$ and, using (b) and (e), $(1,4,5,6,8) \prec(2,3,5,7,8)$.

COROLlaRY. In each special value permutation, the last $2^{n-1}$ elements, in reverse order, are the complements with respect to $N=$ $(1,2, \cdots, n)$ of the first $2^{n-1}$.

This follows from part (d) of the lemma. From a geometric viewpoint, if $J$ and $K$ are complementary, then $p(J)$ and $p(K)$ are opposite vertices of $C$; that is, they are symmetric in $(1 / 2,1 / 2, \cdots, 1 / 2)$. They are therefore equidistant from and separated by the $(n-1)$-plane

$$
\sum v_{i} x_{i}=1 / 2 \sum v_{i}
$$

Therefore, (1) the vertices of $C$ not in $\{p\}_{\Sigma v_{i} / 2}$ are opposite those in $\{p\}_{\Sigma v_{i} / 2}$, and (2) as $c$ increases from 0 to a value exceeding $\sum v_{i}, L^{n-1}(c)$ passes through the vertices not in $\{p\}_{\Sigma v_{i} / 2}$ in the reverse of the order in which it passes through their respective opposite vertices.

(D) This geometric proof implies that the corollary holds for $\Pi\{V,[J]\}$, independently of the hypotheses in $\S 2(\mathrm{E})$.

LEMma 3.3. A permutation $\Pi$ of the elements of $\{J\}$ is a special value permutation if and only if the following inequalities are consistent

(a) $0<v_{1}<\cdots<v_{n}$

(b) $\sum_{j \in J} v_{j}<\sum_{k \in K} v_{k} \quad$ if $J$ precedes $K$ in $\Pi$.

In such case, $\Pi=\Pi(V, J)$ for each value set $V$ satisfying (3.10).

This lemma follows directly from the definitions.

COROLlARY. If $n>1$, each special value permutation commences with $0,1,2$ and terminates with $134 \cdots n, 234 \cdots n, 123 \cdots n$.

4. A recurrency. The cases $n<5$. To distinguish among different values of $n$, we will sometimes use $\left\{\Pi^{n}\right\}$, instead of merely $\{\Pi\}$ to denote the set of all special value permutations of subsets of $N=(1, \cdots, n)$. 
For $n<4$, we have

$$
\left\{\Pi^{1}\right\}: 01
$$

$\left\{\Pi^{2}\right\}: 01212$

$$
\left\{\begin{array}{llllllllll}
\left\{\Pi^{3}\right\}=\Pi_{1}^{3}, \Pi_{2}^{3} & \text { where } & \Pi_{1}^{3}=0 & 1 & 2 & 3 & 12 & 13 & 23 & 123 \\
& & \Pi_{2}^{3}=0 & 1 & 2 & 12 & 3 & 13 & 23 & 123
\end{array}\right.
$$

(A) In studying the relationship between $\left\{\Pi^{n-1}\right\}$ and $\left\{\Pi^{n}\right\}$, we will use the symbol $\Pi^{n}\left(v_{1}, \cdots, v_{n}\right)$ for $\Pi(V, J)$ and $\Pi^{n-1}\left(v_{1}, \cdots, v_{n-1}\right)$ for $\Pi\left(V^{\prime}, J^{\prime}\right)$, where $V^{\prime}=\left(v_{1}, \cdots, v_{n-1}\right)$ and $J^{\prime}$ is generic notation for a subset of $N^{\prime}=(1, \cdots, n-1)$.

LEMma 4.1. The sequence $\Pi^{n-1}\left(v_{1}, \cdots, v_{n-1}\right)$ is obtainable from $\Pi^{n}\left(v_{1}, \cdots, v_{n}\right)$ by deleting from the latter each $J$ which contains the element $n$. The sequence of deleted elements is obtainable, in order, by adjoining $n$ to each $J^{\prime}$ in the sequence $\Pi^{n-1}\left(v_{1}, \cdots, v_{n-1}\right)$.

This follows directly from the definitions of the sequences in terms of the respective value functions defined by $V^{\prime}$ on $\left\{J^{\prime}\right\}$ and by $V$ on $\{J\}$. It also follows geometrically, with the aid of the fact that the plane $L^{n-1}(c)$, defined by (1.10), intersects the $(n-1)$-planes $x_{n}=0$ and $x_{n}=1$, respectively, in a pair of parallel $(n-2)$-planes. The $(n-2)$ plane for $x_{n}=0$ is the $L^{n-2}(c)$ corresponding to $\left(V^{\prime}, J^{\prime}\right)$. Because of the parallelism, the $(n-2)$-plane for $x_{n}=1$ passes through the sequence of vertices corresponding to the deleted elements in the order specified by the lemma.

To illustrate, let

$$
\begin{aligned}
& \Pi^{4}\left(v_{1}, v_{2}, v_{3}, v_{4}\right)=012312413142324123 \\
& 341241342341234 \text {. }
\end{aligned}
$$

Then

$$
\Pi^{3}\left(v_{1}, v_{2}, v_{3}\right)=0123121323123=\Pi_{1}^{3}
$$

and the complementary subsequence of $\Pi^{4}(V)$ is, in accordance with Lemma 4.1,

$$
\Pi^{\prime}=41424341241342341234
$$

(B) Let $\Pi \in\left\{\Pi^{n-1}\right\}$, and let $\Pi^{\prime}$ be obtained from $\Pi$ by adjoining $n$ to each element of the latter; just as $\Pi^{\prime}$ in (4.4) is obtained by adjoining 4 to each element in (4.3). If $\Pi^{*}$ is a permutation of $\{J\}$ with $\Pi, \Pi^{\prime}$ for complementary subsequences, then it will be said that $\Pi^{*}$ is obtained by meshing $\Pi$ and $\Pi^{\prime}$. An admissible meshing is one whose associated 
inequalities (3.10) are consistent; that is, one for which $\Pi^{*} \in\left\{\Pi^{n}\right\}$.

Lemma 4.2. In the notation of $(\mathrm{A})$, let $\Pi^{\prime}$ be admissibly meshed with $\Pi$ in all possible ways for each $\Pi \in\left\{\Pi^{n-1}\right\}$. The resulting permutations $\Pi^{*}$ of $\{J\}$ are all different and constitute the set $\left\{\Pi^{n}\right\}$.

Proof. Two different meshings of $\Pi$ and $\Pi^{\prime}$ obviously yield different permutations. If $\Pi_{1}^{*}$ results from meshing $\Pi_{1}$ with $\Pi_{1}^{\prime}$ and $\Pi_{2}^{*}$ results from meshing $\Pi_{2}$ with $\Pi_{2}^{\prime}$ where $\Pi_{2} \neq \Pi_{1}$, then, obviously, $\Pi_{1}^{*} \neq \Pi_{2}^{*}$. That we obtain all of $\left\{\Pi^{n}\right\}$ follows from Lemma 4.1.

(C) Lemma 4.2 yields a recurrent procedure for passing from $\left\{\Pi^{n-1}\right\}$ to $\left\{\Pi^{n}\right\}$, whose practicability depends on an analysis of admissible meshings.

To illustrate, we obtain $\left\{\Pi^{4}\right\}$ from $\left\{\Pi^{3}\right\}$. We start with $\Pi_{1}^{3}$ and the associated $\Pi^{\prime}$ in (4.4). As a consequence of Lemma 3.2, Corollary, it suffices to write the first 8 terms, instead of all 16, for each meshing. Since $23 \prec 24$ in every $\Pi \in\left\{\Pi^{4}\right\}$, by Lemma $3.2(\mathrm{e})$, the element 24 cannot be in the first half of an admissible meshing. Hence our task reduces to inserting 4 and 14 into $\Pi_{1}^{3}$ in all ways consistent with the definition of admissibility. Since $3 \prec 4$ and $13 \prec 14$, the only conceivable possibilities are

$$
\begin{aligned}
& \Pi_{1}^{4}=\begin{array}{llllllllll}
0 & 1 & 2 & 3 & 4 & 12 & 13 & 14 & \cdots
\end{array} \text { [The second half } \\
& \Pi_{2}^{4}=0 \begin{array}{lllllll}
0 & 1 & 2 & 3 & 4 & 12 & 1323 \ldots \text { of each } \Pi_{i}^{4} \text { is }
\end{array} \\
& \Pi_{3}^{4}=\begin{array}{llllllll}
0 & 1 & 2 & 3 & 12 & 4 & 13 & 14
\end{array} \ldots \text { given by Lemma } \\
& \Pi_{4}^{4}=\begin{array}{llllllllll}
0 & 1 & 2 & 3 & 12 & 4 & 13 & 23 & \ldots & 3.2 \text {, Corollary] }
\end{array} \\
& \Pi_{5}^{4}=\begin{array}{lllllllll}
0 & 1 & 2 & 3 & 12 & 13 & 4 & 14 & \cdots
\end{array} \\
& \Pi_{6}^{4}=\begin{array}{lllllllll}
0 & 1 & 2 & 3 & 12 & 13 & 4 & 23 & \cdots
\end{array} \\
& \Pi_{7}^{4}=\begin{array}{lllllllll}
0 & 1 & 2 & 3 & 12 & 13 & 23 & 4 & \cdots
\end{array} \\
& \Pi_{8}^{4}=\begin{array}{llllllllll}
0 & 1 & 2 & 3 & 12 & 13 & 23 & 123 & \ldots
\end{array}
\end{aligned}
$$

Similarly, the only conceivable possibilities resulting from $\Pi_{2}^{3}$ are

$$
\begin{array}{llllllllll}
\Pi_{9}^{4}= & 0 & 1 & 2 & 12 & 3 & 4 & 13 & 14 & \cdots \\
\Pi_{10}^{4}= & 0 & 1 & 2 & 12 & 3 & 4 & 13 & 23 & \cdots \\
\Pi_{11}^{4}= & 0 & 1 & 2 & 12 & 3 & 13 & 4 & 14 & \cdots \\
\Pi_{12}^{4}= & 0 & 1 & 2 & 12 & 3 & 13 & 4 & 23 & \cdots \\
\Pi_{13}^{4}= & 0 & 1 & 2 & 12 & 3 & 13 & 23 & 4 \\
\Pi_{14}^{4} & 0 & 0 & 1 & 2 & 12 & 3 & 13 & 23 & 123
\end{array}
$$

To verify that all these possibilities are realized, hence that $\left\{\Pi^{4}\right\}$ consists precisely of these fourteen permutations, it suffices to check 
the consistency of a set of defining inequalities among the $v$ 's. Now $\Pi_{1}^{3}$ is determined by each $\left(v_{1}, v_{2}, v_{3}\right)$ such that

$$
0<v_{1}<v_{2}<v_{3}<v_{1}+v_{2} \text {. }
$$

We obtain $\Pi_{1}^{4}$ by imposing on $v_{4}$ the conditions $v_{3}<v_{4}<v_{1}+v_{2}$ and $v_{1}+v_{3}<v_{1}+v_{4}<v_{2}+v_{3}$, or

$$
\left.v_{3}<v_{4}<\min \left(v_{1}+v_{2}, v_{3}+v_{2}-v_{1}\right) \text { [for } \Pi_{1}^{4}\right]
$$

which are clearly consistent with (4.7). For $\Pi_{2}^{4}$, the conditions on $v_{4}$ are $v_{3}<v_{4}<v_{1}+v_{2}$ and $v_{2}+v_{3}<v_{1}+v_{4}$ or

$$
v_{3}+v_{2}-v_{1}<v_{4}<v_{1}+v_{2}
$$

which can be satisfied if and only if

$$
v_{3}<2 v_{1} \text {. }
$$

Proceeding thus with the eight permutations (4.5) we arrive at the following:

\begin{tabular}{l|l|l}
$i$ & $\begin{array}{c}\text { Necessary conditions on } \\
\left(v_{1}, v_{2}, v_{3}\right) \text { for } \Pi_{i}^{4}\end{array}$ & \multicolumn{1}{c}{ Conditions on $v_{4}$ for $\Pi_{i}^{4}$} \\
\hline 1 & $(4.7)$ & $v_{3}<v_{4}<\min \left(v_{1}+v_{2}, v_{2}+v_{3}-v_{1}\right)$ \\
6 & $(4.7)$ & $v_{4}>\max \left(v_{1}+v_{2}, v_{2}+v_{3}-v_{1}\right)$ \\
7 & $(4.7)$ & $v_{2}+v_{3}<v_{4}<v_{1}+v_{2}+v_{3}$ \\
8 & $(4.7)$ & $v_{1}+v_{2}+v_{3}<v_{4}$ \\
2 & $(4.7), v_{3}<2 v_{1}$ & $v_{2}+v_{3}-v_{1}<v_{4}<v_{1}+v_{2}$ \\
3 & $(4.7), v_{3}>2 v_{1}$ & $v_{1}+v_{2}<v_{4}<\min \left(v_{1}+v_{3}, v_{2}+v_{3}-v_{1}\right)$ \\
4 & $(4.7), v_{2}<2 v_{1}$ & $\max \left(v_{1}+v_{2}, v_{2}+v_{3}-v_{1}\right)<v_{4}<v_{1}+v_{3}$ \\
5 & $(4.7), v_{2}>2 v_{1}$ & $v_{1}+v_{3}<v_{4}<v_{2}+v_{3}-v_{1}$
\end{tabular}

In the following similar tabulation for the six permutations (4.6), condition (4.7) is replaced by

$$
0<v_{1}<v_{2}<v_{1}+v_{2}<v_{3}
$$

which is the defining condition for $\Pi_{2}^{3}$.

\begin{tabular}{r|l|l}
\multicolumn{1}{r|}{} & \multicolumn{1}{|c|}{ Necessary for $\Pi_{i}^{4}$} & \multicolumn{1}{|c}{ Condition on $v_{4}$ for $\Pi_{i}^{4}$} \\
\hline 9 & $(4.11)$ & $v_{3}<v_{4}<v_{2}+v_{3}-v_{1}$ \\
12 & $(4.11)$ & $\max \left(v_{1}+v_{3}, v_{2}+v_{3}-v_{1}\right)<v_{4}<v_{1}+v_{2}+v_{3}$ \\
13 & $(4.11)$ & $v_{2}+v_{3}<v_{4}<v_{1}+v_{2}+v_{3}$ \\
14 & $(4.11)$ & $v_{1}+v_{2}+v_{3}<v_{4}$ \\
10 & $(4.11), v_{2}<2 v_{1}$ & $\max \left(v_{3}, v_{2}+v_{3}-v_{1}\right)<v_{4}<v_{1}+v_{3}$ \\
11 & $(4.11), v_{2}>2 v_{1}$ & $v_{1}+v_{3}<v_{4}<v_{2}+v_{3}-v_{1}$
\end{tabular}


Geometrically, $v_{3}<2 v_{1}$, for example, signifies that the 3-plane $L^{3}(c)$, as $c$ increases, passes through the mid point of the edge from $p_{0}:(0,0,0,0)$ to $p_{3}:(0,0,1,0)$ before it passes through the vertex $p_{1}:(1,0,0,0)$, and $v_{3}>2 v_{1}$ means that it passes through these two points in the reverse order. Similar interpretations hold for the conditions $v_{2}<2 v_{1}$ and $v_{2}>$ $2 v_{1}$ in the above tables.

LEMMA 4.3. If the condition $v_{1}<v_{2}<v_{3}<v_{4}$ is dropped, and the condition that $J \neq K$ implies $v(J) \neq v(K)$ is retained, the set $\{\Pi(V, J)\}$ consists of $336=(4 !)(14)$ permutations.

Proof. Case I $\left(v_{i}>0 ; i=1,2,3,4\right)$. In this case, Lemma 4.3 follows from $\S 2$ (B). The 336 permutations can be denoted by $\Pi_{i}^{4}\left(j_{1} j_{2} j_{3} j_{4}\right)$ $(i=1, \cdots, 14)$ as $\left(j_{1}, j_{2}, j_{3}, j_{4}\right)$ ranges over the 24 permutations of $(1,2,3,4)$, where $\Pi_{i}^{4}\left(j_{1} j_{2} j_{3} j_{4}\right)$ is obtained by replacing $h$ by $j_{h}$ in each element of $\Pi_{i}^{4}(h=1,2,3,4)$.

The general case follows from Case I by $\S 2(\mathrm{~B})$.

\section{Applications, interpretations, generalizations.}

LEMmA 5.1. Let $\nu(n)$ denote the number of permutations in the set $\left\{\Pi^{n}\right\}$. If the condition $v_{1}<v_{2}<\cdots<v_{n}$ is dropped, and the condition that $J \neq K$ implies $v(J) \neq v(K)$ is retained, the set $\{\Pi(V, J)\}$ consists of $(n !) \nu(n)$ permutations.

The proof of Lemma 4.3 directly generalizes to Lemma 5.1.

(A) Let $V=\left(v_{1}, \cdots, v_{n}\right)$ and $W=\left(w_{1}, \cdots, w_{n}\right)$ be two special value sets, with $v$ and $w$ denoting the corresponding value functions. Suppose it is desired to find a subset $J$ of $N$ which maximizes $v(J)$ subject to a restriction of the form $w(J)<d$, a constant. Suppose the permutations

(a) $\Pi(V, J)=J_{0}, \cdots, J_{2^{n}-1}$

(b) $\Pi(W, J)=K_{0}, \cdots, K_{2^{n-1}}$

are known. Let $K_{0}, K_{1}, \cdots, K_{\mu}$ be the subsequence of $\Pi(W, J)$ such that $w\left(K_{\mu}\right)<d$ and $w\left(K_{\mu+1}\right) \geqq d$.

Then the $K_{i}(i \leqq \mu)$ which is farthest out in $\Pi(V, J)$ solves the problem. For $n=4$, solutions are thus readily obtained from (4.5) and (4.6). The material in $\S 2$ permits a passage from special to general value sets.

In principle, the method whereby the fourteen sequences $\left\{\Pi^{4}\right\}$ were obtained from the two sequences $\left\{\Pi^{3}\right\}$ can be used to pass from $\left\{\Pi^{n-1}\right\}$ to $\left\{\Pi^{n}\right\}$ for $n=5,6, \cdots$; but it becomes more and more cumbersome as 
$n$ increases.

We next offer some geometric interpretations.

(B) Given a value set $V$, not necessarily special, we will denote by $\Gamma(c)$ the convex hull of the set of points $\{p\}_{c}$, defined in (3.1). Two such convex hulls, corresponding to different value sets $V$ and $W$, are of the same type if they are congruent. From $\$ 2$, it follows that at least one representative of each type is obtained as $V$ ranges over all special value sets and $c$ ranges over the nonnegative reals. A particular $\Gamma(c)$ is carried into all others of the same type by the euclidian transformations of the $n$-cube $C$ onto itself.

THEOREM 5.1. In the case $n=4$, there are exactly 27 types of convex hull $\Gamma(c)$.

Proof. As a consequence of (B), the vertices of each $\Gamma(c)$ correspond to the first $k$ terms $(0 \leqq k \leqq 16)$ of one of the permutations $\Pi_{i}^{4}(i=$ $1, \cdots, 14)$ listed in (4.5) and (4.6). Conversely, each such set of $k$ terms corresponds to the vertices of some $\Gamma(c)$. It is now a simple matter to verify Theorem 5.1 directly, and to list the 27 types: simplexes of dimensions $\leqq 4$, solid $k$-cubes $(k=2,3,4)$, various pyramids and wedges, and so on.

(C) In connection with the maximum value problem suggested in (A), let $\Gamma$ denote the convex hull determined by $w(K)<d$, and let $\Gamma_{0}, \Gamma_{2}, \cdots, \Gamma_{2^{n-1}}$ be the successive convex hulls determined by $v(J)<c$ as $c$ increases. In the sequence of intersections $\Gamma \cap \Gamma_{i}\left(i=0,1, \cdots, 2^{n}-1\right)$, the first is vacuous, since $\Gamma_{0}=\phi$, and the last equals $\Gamma$, since $\Gamma_{2^{n}-1}=$ $C$. Let $\Gamma \cap \Gamma_{j}$ be the first intersection in this sequence which concides with $\Gamma$. If $V$ is a special value set, then $\Gamma \cap \Gamma_{j}$ has just one vertex which is not on $\Gamma \cap \Gamma_{j-1}$, and that vertex corresponds to the solution of the problem. If $V$ is not a special value set, $\Gamma \cap \Gamma_{j}$ may have more than one vertex not on $\Gamma \cap \Gamma_{j-1}$, and each such vertex corresponds to a solution.

(D) Let $C_{0}^{n-1}$ and $C_{1}^{n-1}$ be the two faces of the $n$-cube $C=C^{n}$ on which $x_{n}=0$ and $x_{n}=1$, respectively. Let $\Pi=\Pi(V, J)$ be a special value permutation, and let $\Gamma_{0}(c)$ and $\Gamma_{0}\left(c^{\prime}\right)\left(c^{\prime}>c\right)$ be convex hulls both on $C_{0}^{n-1}$ and both associated with the same permutation $\Pi \in\left\{\Pi^{n-1}\right\}$. Let $\Gamma_{1}(c)$ be the projection of $\Gamma_{0}(c)$ on $C_{1}^{n-1}$. Then (1) the convex hull of $\Gamma_{1}(c) \cup \Gamma_{0}\left(c^{\prime}\right)$ is a $\Gamma(c)$ associated with some permutation $\Pi^{*} \in\left\{\Pi^{n}\right\}$ and (2) a representative of each type of $\Gamma(c)$ is thus obtainable.

The 27 types of $\Gamma(c)$ for $n=4$, together with their associated permutations, thus lead to all the types of $\Gamma(c)$ for $n=5$. To pass 
similarly to $n=6$, we need to know not only the types for $n=5$ but which pairs of them arise from identical permutations.

(E) The following method of linear representation might prove fruitful. For a given special value set $V$, let the $2^{n}$ values $\{v(J) \mid J \subset N\}$ be plotted as points on an $x$-axis, and let $v(J)$ denote both the point and the value. The points are ordered just like the corresponding sets $\{J\}$ in the permutation $\Pi(V, J)$. They constitute a linear representation of $\Pi(V, J)$.

Consider two elements, $J$ and $K$, of $\{J\}$, and let

$$
\begin{aligned}
(J-J \cap K) & =j_{0} \cdots j_{\alpha}\left(0<j_{0}<\cdots<j_{\alpha}\right) \\
(K-J \cap K) & =k_{0} \cdots k_{\beta}\left(0<k_{0}<\cdots<k_{\beta}\right) .
\end{aligned}
$$

The difference

$$
v(K)-v(J)=\left(v_{k_{0}}+\cdots+v_{k_{\beta}}\right)-\left(v_{j_{0}}+\cdots+v_{j_{\alpha}}\right)
$$

measures the directed distance $\overrightarrow{v(J)} \overrightarrow{v(K)}$. This difference will be called essentially positive, essentially negative or indeterminate according as the condition

$$
0<v_{1}<\cdots<v_{n}
$$

implies that it is positive, implies that it is negative, or is consistent with both possibilities. These three cases respectively imply that $J \prec K$ or that $K \prec J$ in every $\Pi \in\{\Pi\}$, or that both possibilities are realized among the permutations $\{\Pi\}$.

\section{LEMMA 5.2. Let}

$$
J \cup K=\left(i_{0}, \cdots, i_{\alpha+\beta+\gamma}\right) \quad 0<i_{0}<\cdots<i_{\alpha+\beta+\gamma} .
$$

If (1) for each $h,\left(i_{h}, \cdots, i_{\alpha+\beta+\gamma}\right)$ contains at least as many k's as $j$ 's, then $v(K)-v(J)$ is essentially positive. If $(2)$ for each $h,\left(i_{h}, \cdots, i_{\alpha+\beta+\gamma}\right)$ contains at least as many $j$ 's as $k$ 's then $v(K)-v(J)$ is essentially negative. If (3) $h, h^{\prime}$ exist such that $\left(i_{h}, \cdots, i_{\alpha+\beta+\gamma}\right)$ contains more $k$ 's than $j$ 's and $\left(i_{h}, \cdots, i_{\alpha+\beta+\gamma}\right)$ contains more $j$ 's than $k$ 's, then $v(K)-v(J)$ is indeterminate.

Proof. Condition (1) implies that $\beta \geqq \alpha$. It also implies that $\left(k_{\beta}>j_{\alpha}\right),\left(k_{\beta-1}>j_{\alpha-1}\right), \cdots,\left(k_{\beta-\alpha}>j_{0}\right)$. Hence,

$$
v(K)-v(J)=\sum_{i=0}^{\alpha}\left(v_{k_{\beta-i}}-v_{j_{\alpha-i}}\right)+\sum_{i=0}^{\beta-\alpha-1} v_{k_{i}}>0 .
$$

Similarly, condition (2) implies $\alpha \geqq \beta$ and $\left(j_{\alpha}>k_{\beta}\right), \cdots,\left(j_{\alpha-\beta}>k_{0}\right)$, so that 


$$
v(K)-v(J)=\sum_{i=0}^{\beta}\left(v_{k_{\beta-i}}-v_{j_{\alpha-i}}\right)-\sum_{i=0}^{\alpha-\beta-1} v_{j_{i}}<0 .
$$

Now assume condition (3) and suppose $k_{\beta}>j_{\alpha}$ so that $v_{k_{\beta}}-v_{j_{\alpha}}>0$. Then $v(K)-v(J)$ can be made positive merely by adding a sufficiently large $w>0$ to each $v_{i}(i \geqq j)$. This increases the first term of (5.6) or (5.7) by $w$, without changing the value of any subsequent term. How, still supposing $k_{\beta}>j_{\alpha}$, let $h$ be the largest integer such that $\left(i_{h}, \cdots, i_{\alpha+\beta+\gamma}\right)$ contains more $j$ 's than $k$ 's. Then $\left(i_{h+1}, \cdots, i_{\alpha+\beta+\gamma}\right)$ contains exactly as many $j$ 's as $k$ 's. Also, the first $(\alpha+\beta+\gamma-h)$ terms of (5.6) or (5.7) are essentially positive, while the next term, $\left(v_{k_{s}}-v_{j_{t}}\right)$ for some $s, t$ or $-v_{\beta_{\beta-\alpha-1}}$, is essentially negative. By adding a sufficiently large $w$ to $v_{i}\left(i \geqq j_{t}\right)$, we can make $\left(v_{k_{s}}-v_{j_{t}}\right)<0$ or $-v_{j_{t}}$ as numerically large as desired, without changing any other terms of (5.6) or (5.7). The proof is easy to complete.

The analysis commenced in this paper could be continued in various ways. The author is publishing it as it stands with the thought that others may wish to refiine and extend it.

UNIVERSITY OF ILLINOIS 


\title{
PACIFIC JOURNAL OF MATHEMATICS
}

\author{
EDITORS
}

Ralph S. Phillips

Stanford University

Stanford, California

M. G. Arsove

University of Washington

Seattle 5, Washington
A. L. Whiteman

University of Southern California Los Angeles 7, California

Lowell J. Paige

University of California

Los Angeles 24, California

\section{ASSOCIATE EDITORS}
E. F. BECKENBACH
D. DERRY
M. OHTSUKA
H. L. ROYDEN
E. SPANIER
E. G. STRAUS
T. M. CHERRY
F. WOLF

\section{SUPPORTING INSTITUTIONS}

\author{
UNIVERSITY OF BRITISH COLUMBIA \\ CALIFORNIA INSTITUTE OF TECHNOLOGY \\ UNIVERSITY OF CALIFORNIA \\ MONTANA STATE UNIVERSITY \\ UNIVERSITY OF NEVADA \\ NEW MEXICO STATE UNIVERSITY \\ OREGON STATE UNIVERSITY \\ UNIVERSITY OF OREGON \\ OSAKA UNIVERSITY \\ UNIVERSITY OF SOUTHERN CALIFORNIA
}

\author{
STANFORD UNIVERSITY \\ UNIVERSITY OF TOKYO \\ UNIVERSITY OF UTAH \\ WASHINGTON STATE UNIVERSITY \\ UNIVERSITY OF WASHINGTON \\ $*$
AMERICAN MATHEMATICAL SOCIETY \\ CALIFORNIA RESEARCH CORPORATION \\ SPACE TECHNOLOGY LABORATORIES \\ NAVAL ORDNANCE TEST STATION
}

Mathematical papers intended for publication in the Pacific Journal of Mathematics should be typewritten (double spaced), and the author should keep a complete copy. Manuscripts may be sent to any one of the four editors. All other communications to the editors should be addressed to the managing editor, L. J. Paige at the University of California, Los Angeles 24, California.

50 reprints per author of each article are furnished free of charge; additional copies may be obtained at cost in multiples of 50 .

The Pacific Journal of Mathematics is published quarterly, in March, June, September, and December. Effective with Volume 13 the price per volume (4 numbers) is $\$ 18.00$; single issues, $\$ 5.00$. Special price for current issues to individual faculty members of supporting institutions and to individual members of the American Mathematical Society: $\$ 8.00$ per volume; single issues $\$ 2.50$. Back numbers are available.

Subscriptions, orders for back numbers, and changes of address should be sent to Pacific Journal of Mathematics, 103 Highland Boulevard, Berkeley 8, California.

Printed at Kokusai Bunken Insatsusha (International Academic Printing Co., Ltd.), No. 6, 2-chome, Fujimi-cho, Chiyoda-ku, Tokyo, Japan.

PUBLISHED BY PACIFIC JOURNAL OF MATHEMATICS, A NON-PROFIT CORPORATION

The Supporting Institutions listed above contribute to the cost of publication of this Journal, but they are not owners or publishers and have no responsibility for its content or policies. 


\section{Pacific Journal of Mathematics}

\section{Vol. 12, No. $2 \quad$ February, 1962}

William George Bade and Robert S. Freeman, Closed extensions of the Laplace operator determined by a general class of boundary conditions . . . . . . . 395

William Browder and Edwin Spanier, H-spaces and duality ............. 411

Stewart S. Cairns, On permutations induced by linear value functions . . . . . . . 415

Frank Sydney Cater, On Hilbert space operators and operator roots of

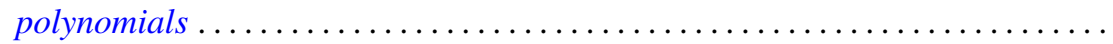

Stephen Urban Chase, Torsion-free modules over $K[x, y] \ldots \ldots \ldots \ldots \ldots \ldots \ldots 437$

Heron S. Collins, Remarks on affine semigroups . . . . . . . . . . . . . . . . 449

Peter Crawley, Direct decompositions with finite dimensional factors . . . . . . . 457

Richard Brian Darst, A continuity property for vector valued measurable

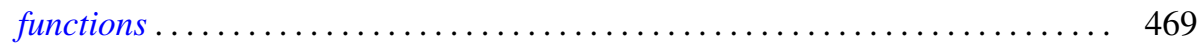

R. P. Dilworth, Abstract commutative ideal theory ................. 481

P. H. Doyle, III and John Gilbert Hocking, Continuously invertible spaces . . . . . . 499

Shaul Foguel, Markov processes with stationary measure . . . . . . . . . . . 505

Andrew Mattei Gleason, The abstract theorem of Cauchy-Weil ............ 511

Allan Brasted Gray, Jr., Normal subgroups of monomial groups . . . . . . . . . . 527

Melvin Henriksen and John Rolfe Isbell, Lattice-ordered rings and function

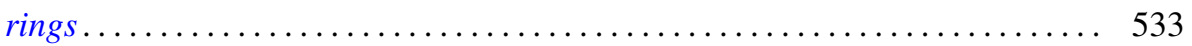

Amnon Jakimovski, Tauberian constants for the $[J, f(x)]$ transformations . ..... 567

Hubert Collings Kennedy, Group membership in semigroups . . . . . . . . . . . 577

Eleanor Killam, The spectrum and the radical in locally $m$-convex algebras ..... 581

Arthur H. Kruse, Completion of mathematical systems . . . . . . . . . . . . . 589

Magnus Lindberg, On two Tauberian remainder theorems ................ 607

Lionello A. Lombardi, A general solution of Tonelli's problem of the calculus of

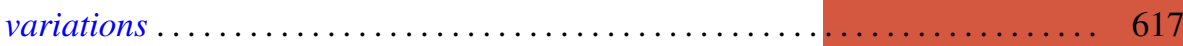

Marvin David Marcus and Morris Newman, The sum of the elements of the powers

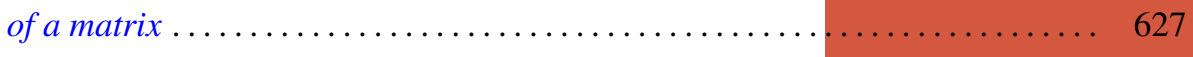

Michael Bahir Maschler, Derivatives of the harmonic measures in

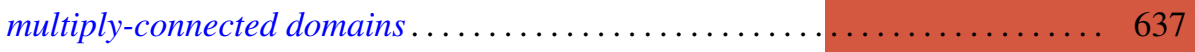

Deane Montgomery and Hans Samelson, On the action of $\mathrm{SO}(3)$ on $S^{n} \ldots \ldots \ldots 649$

J. Barros-Neto, Analytic composition kernels on Lie groups . . . . . . . . . . . . 661

Mario Petrich, Semicharacters of the Cartesian product of two semigroups ...... 679

John Sydney Pym, Idempotent measures on semigroups . . . . . . . . . . . . 685

K. Rogers and Ernst Gabor Straus, A special class of matrices . . . . . . . . . . . . 699

U. Shukla, On the projective cover of a module and related results . . . . . . . . . 709

Don Harrell Tucker, An existence theorem for a Goursat problem . . . . . . . . . . . 719

George Gustave Weill, Reproducing kernels and orthogonal kernels for analytic

differentials on Riemann surfaces ......................... 729

George Gustave Weill, Capacity differentials on open Riemann surfaces ........ 769

G. K. White, Iterations of generalized Euler functions . . . . . . . . . . . . . 777

Adil Mohamed Yaqub, On certain finite rings and ring-logics . . . . . . . . . 785 